\title{
INTERVENÇÃO EDUCATIVA SOBRE TUBERCULOSE PARA AGENTES COMUNITÁRIOS DE SAÚDE EM UNIDADES DA ATENÇÃO PRIMÁRIA À SAÚDE EM UM MUNICÍPIO DA REGIÃ̃O NORTE ${ }^{1}$
}

Educational intervention on tuberculosis for community health workers in Primary Health Care units in a municipality of the North Region

Intervención educativa sobre tuberculosis para agentes comunitarios de salud en unidades de Atención Primaria de Salud en un municipio de la Región Norte

\section{Pollyana Roberta Freitas*1, Eduardo Rezende Honda ${ }^{2}$, Erika Simone Galvão Pinto ${ }^{3}$, Melisane Regina Lima Ferreira ${ }^{4}$, Nathalia Halax Orfão ${ }^{5}$}

${ }^{1}$ Enfermeira, Mestre em Ensino em Ciências da Saúde, Secretaria de Estado da Saúde de Rondônia, Porto Velho, Rondônia, Brasil

${ }^{2}$ Farmacêutico, Pós Doutor em Biologia Geral, Laboratório Central de Saúde Pública de Rondônia, Porto Velho, Rondônia, Brasil

${ }^{3}$ Enfermeira, Doutora em Ciências, Universidade Federal do Rio Grande do Norte, Natal, Rio Grande do Norte, Brasil ${ }^{4}$ Enfermeira, Pós-graduanda em Especialização em Saúde Pública, Universidade Federal de Rondônia, Porto Velho, Rondônia, Brasil

${ }^{5}$ Enfermeira, Doutora em Ciências, Universidade Federal de Rondônia, Porto Velho, Rondônia, Brasil

*Correspondência: Universidade Federal de Rondônia, Campus José Ribeiro Filho - BR 364, Km 9,5. Porto VelhoRO, Brasil. CEP: 76.801-059. E-mail: pollyrobe@gmail.com

Artigo recebido em 02/03/2020 aprovado em 10/06/2020 publicado em 29/08/2020.

\section{RESUMO}

Diante da necessidade de propor uma qualificação para transformação e aprimoramento dos Agentes Comunitários de Saúde (ACS) na atenção à tuberculose (TB), este estudo buscou desenvolver uma intervenção educativa sobre TB para os ACS, de duas Unidades de Saúde da Família, no município de Porto Velho-RO. Trata-se de um estudo de intervenção baseado em dados obtidos de forma descritiva por meio de um questionário estruturado e analisado através de estatística descritiva, a partir da categorização das respostas em adequadas/suficientes e não adequadas/insuficientes. A intervenção foi delineada, a partir das lacunas do conhecimento identificadas, e com base no desenvolvimento de atividades em grupo realizada em encontros de quatro horas cada, com os mesmos conteúdos e metodologia, para 14 ACS da U1 (dois períodos) e 10 da U2 (um período). Verificou-se um conhecimento restrito sobre a doença, e a partir da intervenção educativa com apresentação individual, dinâmica de grupo, discussão de texto, exposição dialogada e uma avaliação, a qual os participantes empregaram o termo "ótima" para a metodologia utilizada. Tais achados permitem refletir sobre a importância dos processos formativos e de qualificações que possibilitem que os profissionais possam dialogar sobre o cenário em que suas atribuições são desempenhadas e as dificuldades vivenciadas.

Palavras-chave: Tuberculose; Agentes Comunitários de Saúde; Atenção Primária à Saúde; Educação em Saúde;

Serviços de Saúde.

\section{ABSTRACT}

\footnotetext{
${ }^{1}$ Artigo da dissertação de mestrado intitulada "Intervenção sobre tuberculose para agentes comunitários de saúde no município de Porto Velho-RO” apresentada ao Programa de Mestrado Profissional em Ensino em Ciências da Saúde da Universidade Federal de Rondônia (UNIR).
} 
In view of the need to propose a qualification for the transformation and improvement of Community Health Workers (CHW) in attention to Tuberculosis (TB). Thus, the present study aimed to develop an educational intervention on TB for CHW, from two Family Health Units, in the city of Porto Velho-RO. This is an intervention study based in descriptive data using a structured questionnaire and analyzed using descriptive statistics, from the categorization of responses into adequate / sufficient and not adequate / insufficient. The intervention was designed based on the knowledge gaps identified and on the development of group activities carried out in meetings of four hours each, with the same content and methodology for 14 CHW from U1 (two periods) and 10 from U2 (one period). It was found that the CHW had limited knowledge about TB and the educational intervention with individual presentation, group dynamics, text discussion, dialogued exposition and an evaluation which the participants used the term "excellent" for the methodology used. Such findings allow us to reflect on the importance of the training processes and qualifications that enable the dialogue with the professionals about the scenario in which their duties are performed and the difficulties experienced.

Keywords: Tuberculosis; Community Health Workers; Primary Health Care; Health Education; Health Services.

\section{RESUMEN}

En vista de la necesidad de proponer una calificación para la transformación y mejora de los Agentes Comunitarios de Salud (CHA en atención a Tuberculosis (TB). Por lo tanto, el presente estudio tuvo como objetivo desarrollar una intervención educativa sobre TB para CHA, de dos Unidades de Salud Familiar, en la ciudad de Porto Velho-RO. Este es un estudio de intervención basado en datos obtenidos descriptivamente a través de un cuestionario estructurado y un análisis utilizando estadísticas descriptivas de la categorización de las respuestas en adecuada $/$ suficiente y no adecuada / insuficiente. La intervención fue diseñada en base a las brechas de conocimiento identificadas y basado en el desarrollo de actividades grupales realizadas en reuniones de cuatro horas cada una, con el mismo contenido y metodología, para 14 CHA de U1 (dos períodos) y 10 de U2 (un período). Se descubrió que la CHA tenía un conocimiento limitado sobre la TB y basado en la intervención educativa con presentación individual, dinámica de grupo, discusión de texto, exposición dialogada y una evaluación, que los participantes usaron el término "excelente" para la metodología utilizada. Tales hallazgos nos permiten reflexionar sobre la importancia de los procesos de capacitación y las calificaciones que permiten a los profesionales discutir el escenario en el que se desempeñan sus funciones y las dificultades experimentadas.

Descriptores: Tuberculosis; Agentes Comunitarios de Salud; Atención Primaria de Salud; Educación en Salud; Servicios de Salud.

\section{INTRODUÇÃO}

A Tuberculose (TB) é uma doença infecciosa existente desde as civilizações mais antigas. Em 2018, estimou-se que 10 milhões de pessoas foram acometidas pela doença, e que 1,2 milhões de pessoas no mundo foram a óbito tendo a TB como causa principal, ultrapassando os óbitos por HIV e malária, juntos (WHO, 2019).

No Brasil, em 2018, em relação ao desfecho do tratamento da doença não foram alcançadas as metas estabelecidas pela Organização Mundial da Saúde (OMS), observando-se baixa taxa de cura $(71,4 \%)$ e elevada de abandono $(10,8 \%)$, considerando o preconizado de pelos menos $85 \%$ e no máximo $5 \%$, respectivamente (BRASIL, 2019).
Nesse cenário, a Atenção Primária à Saúde (APS), alicerce central do Sistema Único de Saúde (SUS), figura como principal aliada na luta contra a doença, principalmente por meio da Estratégia de Saúde da Família (ESF), tendo em vista que a diminuição da incidência e do abandono do tratamento da TB estão intimamente relacionados com a cobertura da ESF e o desenvolvimento de suas atribuições (BRASIL, 2017).

A ESF é desenvolvida através de práticas interdisciplinares a um número definido de famílias, em uma área geográfica delimitada, por equipes multiprofissionais, cujo trabalho se inicia por meio das atividades dos Agentes Comunitários de Saúde (ACS) que representam um elo entre o serviço e a comunidade (FRANCO e MERHY, 2007), baseado 
em ações de promoção da saúde junto às famílias, prevenção de doenças e vigilância em saúde através das Visitas Domiciliárias (VD) e ações educativas (MELO et al., 2015).

$\mathrm{Na}$ atenção à TB, o ACS tem o papel de identificar os sintomáticos respiratórios (SR) nos domicílios e na comunidade e encaminhá-los juntamente com os contatos para avaliação nas unidades de saúde. Além disso, o ACS deve orientar quanto à importância da realização dos exames diagnósticos e a coleta/encaminhamento do escarro para análise laboratorial, bem como supervisionar a tomada de medicação e realizar a busca de faltosos e de pacientes que abandonaram o tratamento (BRASIL, 2011a; BRASIL, 2011b).

Quando tais atividades são desempenhadas, elas podem contribuir na adesão ao tratamento da TB, além de se caracterizarem como essenciais para vigilância em saúde, na medida em que podem identificar situações de vulnerabilidade à doença, principalmente no contexto social de sua microárea (SOUZA et al., 2014). Contrariamente, o desconhecimento do ACS quanto as suas atribuições e a falta de qualificação técnica desses profissionais sobre a temática (SÁ et al., 2011) podem comprometer a continuidade do cuidado e o controle da doença (CARDOZO-GONZALES et al., 2015).

$\mathrm{Na}$ perspectiva do SUS, as dificuldades operacionais do trabalho do ACS estão relacionadas à falta de formação adequada para o desenvolvimento de suas atribuições direcionadas às necessidades de saúde da população (CARDOZO-GONZALES et al., 2015).

No entanto, em face da importância e complexidade das ações do ACS na detecção precoce dos suspeitos de TB na comunidade e o manejo dos casos da doença no âmbito da APS (CRISPIM et al., 2012), é essencial que ações educativas sejam desenvolvidas com base na realidade prática e nas necessidades destes profissionais, que superem os limites da discussão teórica e conceitual da atuação do ACS (GOUVÊA et al., 2015).

Assim, diante da necessidade de propor uma qualificação para transformação e aprimoramento desses profissionais, considera-se de fundamental importância a identificação do conhecimento desses atores. Para tanto, o presente estudo teve como objetivo desenvolver uma intervenção educativa sobre TB para os ACS, de duas Unidades de Saúde da Família (USF), no município de Porto Velho-RO, a partir do conhecimento prévio dos ACS sobre TB.

\section{MATERIAIS E MÉTODOS}

Trata-se de um estudo de intervenção baseado em dados obtidos de forma descritiva desenvolvido no município de Porto Velho, capital do estado de Rondônia. Em 2015, entre os estados da Região Norte, Porto Velho foi a terceira capital com maior número de casos notificados de TB (428 casos), o equivalente a 63,2\% do total de notificações no Estado (677 casos). A taxa de incidência da TB no município foi de 51,3 casos por 100 mil habitantes, cujo percentual de cura foi de 70,5\%, e mesmo com a crescente expansão da ESF foi registrado um alto percentual de abandono $(15,7 \%)$ (BRASIL, 2017).

Assim, frente a presente realidade e, considerando que os resultados do controle da doença são determinados, principalmente, pelas ações da ESF, para este estudo foram selecionadas duas USF, sendo uma da Zona 3 (sul) e uma da Zona 2 (norte) do município, as quais foram denominadas de U1 e U2, respectivamente. A seleção dessas unidades ocorreu de forma intencional por apresentarem maior número de notificação de casos novos de TB no ano de 2015 (14 e 12 casos, respectivamente) dentre as USF do município.

Em cada uma das unidades selecionadas, atuavam quatro equipes de saúde da família, sendo essas distribuídas, igualmente, nos períodos matutino 
e vespertino. As áreas de abrangência das equipes eram distintas, ainda que estivessem inseridas em uma única estrutura física. Cada equipe era composta por um enfermeiro, um médico, um cirurgião dentista e um auxiliar/técnico de enfermagem. A U1 contava com 28 ACS e quatro Auxiliares de Saúde Bucal (ASB), enquanto a U2 contava com 16 ACS e quatro ASB.

A população do estudo foi composta por todos os ACS que atuavam nas equipes das USF selecionadas. Foram considerados como critérios de inclusão, os ACS que atuavam na atual equipe de saúde da família há mais de um ano. E como exclusão, os ACS que no período da coleta de dados estavam de licença e/ou férias ou afastados de suas atividades profissionais por qualquer outro motivo, bem como aqueles que, após três tentativas para a entrevista, não foram localizados ou não compareceram aos encontros previamente agendados.

Do total de 44 ACS, 20 foram excluídos, tendo em vista que nove ACS eram recém-contratados pela Administração Pública e por este motivo estavam na atual equipe de saúde da família há menos de um ano; sete ACS não compareceram nas atividades em área na qual foi agendada a realização das entrevistas e/ou não foram localizados nas USF selecionadas após três tentativas; e quatro ACS se encontravam afastados do serviço. Neste sentido, foram entrevistados 24 ACS, sendo 14 da U1 e 10 da U2.

Os dados foram coletados através de um questionário estruturado, elaborado com base no "Manual de Recomendações para o Controle da Tuberculose no Brasil" (BRASIL, 2011a), manual "Tratamento Diretamente Observado da Tuberculose na Atenção Básica: Protocolo de Enfermagem" (BRASIL, 2011b) e guia "Advocacia, Comunicação e Mobilização Social para o Controle da TB: Um Guia para o Desenvolvimento de Pesquisas de Conhecimento, Atitudes e Práticas" (OMS, 2008).
O instrumento foi enviado para avaliação de conteúdo para 10 especialistas na temática e posteriormente realizado teste piloto com seis ACS de outras USF do município, os quais não foram incluídos na população deste estudo. $\mathrm{O}$ questionário foi constituído por 38 questões, com categorias de respostas abertas e fechadas, organizadas em três seções: identificação dos entrevistados, conhecimentos dos ACS sobre TB e autoavaliação do conhecimento.

A coleta de dados ocorreu por meio de entrevistas, entre março e abril de 2017, com tempo médio de 20 minutos, em locais que resguardavam a privacidade dos entrevistados, mediante assinatura do Termo de Consentimento Livre e Esclarecido.

A análise de dados foi realizada com base na categorização das repostas do questionário como adequadas/suficientes e não adequadas/insuficientes. Os resultados obtidos das questões, cuja resposta correta dependia da menção de uma palavra ou expressão-chave, foram categorizados como adequadas ou não adequadas pela presença ou ausência da mesma. Enquanto que aqueles, cuja resposta correta dependia da menção de um conjunto de informações, foram categorizados como suficientes ou insuficientes pela menção das informações consideradas mínimas para o tema. Os dados foram inseridos e analisados através de estatística descritiva em planilha eletrônica (Excel 2011®), validados por dupla digitação.

Para orientar a condução da intervenção, foi estruturado um plano de trabalho com a descrição das atividades previstas. A organização e logística ocorreram em junho de 2017, e incluíram: a elaboração do material pedagógico (apresentação de slides) com base nas lacunas do conhecimento identificadas nas entrevistas com os ACS; a produção do material de apoio; os agendamentos prévios com cada equipe das USF selecionadas; e as providências quanto à 
disponibilização de estrutura física para a realização dos encontros propostos.

A intervenção foi delineada com base no desenvolvimento de atividades em grupo, com apresentação em plenária, cujo objetivo era estimular a integração dos participantes. Para refletir sobre a importância do trabalho em equipe foi proposta a dinâmica "Jogo dos Quadrados", realizada através da montagem de quatro quebra-cabeças.

Em seguida, propôs-se a leitura do texto "O trabalho de Gisele", sobre o manejo de um caso de TB, ressaltando a importância do papel do ACS no controle da doença, para promover um momento de reflexão entre os participantes. As discussões sobre o caso foram incitadas por questões disparadoras, elaboradas com base nas informações contidas no texto e no conteúdo a ser explanado na exposição dialogada em seguida. Ao final, realizou-se uma avaliação individual escrita, momento importante para manifestação das opiniões dos participantes sobre a metodologia adotada e o conteúdo abordado.

O plano de trabalho e o material de apoio foram submetidos à apreciação, adequação e aprovação da coordenação municipal do Programa de Controle da TB, que recomendou a realização da intervenção educativa em encontros, de quatro horas cada, apenas para os ACS da USF selecionadas diante dos resultados encontrados sobre o conhecimento destes sobre TB. Além disso, a coordenação disponibilizou folders informativos sobre a TB e cópias das fichas utilizadas para notificação, exames e acompanhamento, bem como dois livros de Registro do Sintomático Respiratório no Serviço de Saúde, para serem entregues às USF selecionadas após a intervenção.

Após o planejamento da intervenção, foram realizadas duas visitas à U1 e três à U2, entre os meses de junho e julho de 2017, para apresentação dos resultados parciais às enfermeiras de cada equipe $\mathrm{e}$ diretoras das unidades. Posteriormente, foram definidos dois encontros, na mesma data, para os ACS da U1 (um matutino e outro vespertino), e um encontro no período matutino para todos os ACS da U2, conforme solicitação pela direção da referida unidade, com quatro horas cada.

Nos encontros com os profissionais da U1, participaram 30 profissionais, desses 27 eram ACS, dois eram enfermeiras e uma farmacêutica, essas solicitaram à direção da USF a participação como ouvintes devido ao interesse no tema. No encontro com os profissionais da U2, participaram 13 ACS da USF.

O número de ACS participantes da intervenção foi expressivamente superior ao número de entrevistas realizadas para coleta de dados. No entanto, cabe ressaltar que os critérios de exclusão não foram aplicados nesta etapa do estudo, pois todos os ACS das USF selecionadas foram convidados a participar da intervenção, independente de terem participado da coleta de dados do estudo.

Os locais para a realização da intervenção foram selecionados, seguindo critérios de maior proximidade da USF e disponibilidade de espaço físico adequado, sendo estes uma Biblioteca Pública Municipal, localizada a menos de 500 metros de distância da U1 e uma Escola Estadual de Ensino Fundamental e Médio ao lado da U2. Em seguida, foi formalizada a solicitação quanto à cedência do espaço físico.

Foi entregue a cada equipe de saúde da família, com 15 dias de antecedência às datas propostas para intervenção, um convite formal intitulado "Vamos falar sobre Tuberculose?" direcionado aos ACS, informando data, local e hora de cada encontro, sendo também disponibilizado em formato digital, conforme solicitação das diretoras das unidades, para envio aos ACS via aplicativo de conversas online. 
Atendendo a Resolução do Conselho Nacional de Saúde no 466, de 12 de dezembro de 2012, que regulamenta as pesquisas envolvendo seres humanos (BRASIL, 2012), o projeto do presente estudo foi submetido à apreciação e aprovado, pelo CEP da UNIR, conforme Parecer Consubstanciado $\mathrm{n}^{\circ}$ 1.849 .736

\section{RESULTADOS E DISCUSSÃO}

O perfil dos ACS identificado neste estudo (Tabela 1) difere dos encontrados na literatura apenas com relação à faixa etária predominante (41 a 50 anos), superior à encontrada em outros estudos (30 a 40 anos). Ressalta-se que a maioria dos ACS eram casados, o que pode ser um indicador de permanência na microárea onde moram e trabalham, com nível médio de escolaridade, baixa rotatividade dos ACS nas equipes, ainda que o número de famílias seja maior do que o recomendado (DENTI et al., 2014; FREITAS et al., 2015; GARCIA et al., 2017; GUIMARÃES et al., 2017).

Tais achados permitem a reflexão sobre a existência de sobrecargas de trabalho, comprometendo à garantia da realização de visitas mensalmente a todas as famílias (DENTI et al., 2014), estabelecimento e fortalecimento de vínculo, promoção de ações educativas, preventivas, orientações e a identificação de novas demandas (CRISPIM et al., 2012; LANZONI et al., 2014).

No contexto do controle da TB, estudos constataram que, em algumas situações, a visita acontecia de forma mecanizada (CLEMENTINO e MIRANDA, 2015), e que as orientações relacionadas à doença eram direcionadas para casos de TB já confirmados, restringindo-se à tomada da medicação (SÁ et al., 2011).

Tabela 1. Distribuição do perfil dos Agentes Comunitários de Saúde das duas Unidades de Saúde da Família selecionadas, Porto Velho, 2017

\begin{tabular}{|c|c|c|c|}
\hline \multicolumn{2}{|c|}{ Variáveis } & \multirow{2}{*}{$\frac{\mathrm{N}=\mathbf{2 4}}{9}$} & \multirow{2}{*}{$\frac{\%}{37,50}$} \\
\hline \multirow{3}{*}{ Faixa Etária } & $30-40$ & & \\
\hline & $41-50$ & 12 & 50,00 \\
\hline & Acima de 50 & 3 & 12,50 \\
\hline \multirow{2}{*}{ Sexo } & Feminino & 20 & 83,33 \\
\hline & Masculino & 4 & 16,67 \\
\hline \multirow{4}{*}{ Estado Conjugal } & Casado(a) & 10 & 41,67 \\
\hline & Solteiro(a) & 9 & 37,50 \\
\hline & União Estável & 4 & 16,67 \\
\hline & Separado(a)/Divorciado(a) & 1 & 4,17 \\
\hline \multirow{3}{*}{ Formação } & Ensino Médio & 16 & 66,67 \\
\hline & Curso Técnico & 1 & 4,17 \\
\hline & Ensino Superior Completo & 7 & 29,17 \\
\hline \multirow{3}{*}{ Anos de trabalho como ACS } & 1 a 5 & 2 & 8,33 \\
\hline & 6 a 10 & 12 & 50,00 \\
\hline & Acima de 10 & 10 & 41,67 \\
\hline \multirow{3}{*}{ Anos de trabalho na atual ESF } & 1 a 5 & 8 & 33,33 \\
\hline & 6 a 10 & 9 & 37,50 \\
\hline & Acima de 10 & 7 & 29,17 \\
\hline Número de famílias na microárea & Até 150 & 8 & 33,33 \\
\hline
\end{tabular}




\begin{tabular}{|c|c|c|c|}
\hline & 151 a 160 & 10 & 41,67 \\
\hline & 161 a 170 & 4 & 16,67 \\
\hline & Acima de 170 & 2 & 8,33 \\
\hline \multirow{2}{*}{ Portadores de TB na sua microárea } & Não & 23 & 95,83 \\
\hline & Sim & 1 & 4,17 \\
\hline \multirow{2}{*}{ Acompanhou algum caso de TB } & Sim & 13 & 54,17 \\
\hline & Não & 11 & 45,83 \\
\hline \multirow{3}{*}{ Participou de treinamentos sobre TB } & Nenhum & 3 & 12,50 \\
\hline & 1 a 5 & 19 & 79,17 \\
\hline & 6 a 10 & 2 & 8,33 \\
\hline \multirow{3}{*}{ Recebeu materiais didáticos sobre TB } & Nenhum & 6 & 25,00 \\
\hline & 1 a 5 & 14 & 58,33 \\
\hline & 6 a 10 & 4 & 16,67 \\
\hline
\end{tabular}

Legenda: ACS (Agente Comunitário de Saúde). ESF (Estratégia Saúde da Família).

De maneira geral, os achados deste estudo demonstraram que os ACS possuíam um conhecimento restrito sobre a doença (Tabela 2). Destaca-se que a tosse persistente por três ou mais semanas não foi mencionada como sintomatologia da TB pelos entrevistados, sendo este o sintoma mais característico da doença e fundamental para a identificação do SR (BRASIL, 2011a). Uma detecção de casos ineficiente atrasa o diagnóstico do agravo, resultando na instalação do processo de adoecimento e na continuidade da cadeia de transmissão a outras pessoas (VILLA et al., 2013).

Diversos fatores podem resultar na baixa suspeição de casos de TB, levantando questionamentos para futuras investigações. Dentre eles, a falta de treinamento específico sobre a doença (BORNSTEIN e DAVID, 2014) e as falhas no processo de qualificação que, na maioria das vezes, são insuficientes para desenvolver as competências necessárias para o adequado desempenho das atribuições dos ACS (TOMAZ, 2002).

Talvez a escassez de discussões sobre o potencial do ACS para exercer seu papel educador (DURÃO et al., 2011), além da formação, treinamento e capacitação oferecidos a esses profissionais, sejam os limitantes tanto no campo teórico e conceitual do trabalho, como no detalhamento de suas atribuições (SÁ et al., 2011).

Nesse sentido, ações educativas que considerem não apenas os aspectos clínicos da TB, mas também as necessidades que emergem do cotidiano e o conhecimento prévio destes profissionais em relação à temática (CARDOZO-GONZALES et al., 2015; QUEIROZ et al., 2014; FREITAS et al., 2015) serão capazes de promover maior desenvolvimento das habilidades e domínio do conhecimento técnico-científico.

Igualmente preocupante foi o predomínio de respostas inadequadas no que concerne à transmissão, o desconhecimento do conceito e tratamento da Infecção Latente da TB (ILTB), bem como a correta definição dos contatos. Conhecer a cadeia de transmissão e sua relação com as pessoas que convivem com o doente de TB é de grande importância para prevenir o adoecimento e diagnosticar precocemente casos de doença não ativa (LIMA et al., 2013).

Ademais, o ACS inserido na comunidade é um importante facilitador quanto à abordagem e o acompanhamento efetivo dos contatos. No entanto, isso pressupõe que este profissional esteja munido de conhecimento para identificá-los e capacitado para 
realizar o procedimento de investigação, de acordo com os protocolos do Ministério da Saúde (LIMA et al., 2013).

A qualificação de um dos principais atores na efetivação do Tratamento Diretamente Observado (TDO) pode promover a ampliação do acesso aos serviços de saúde (CRISPIM et al., 2012), a aceitabilidade e a adesão ao tratamento, bem como o reconhecimento dos faltosos para intervenções em momento oportuno (BRASIL, 2011b).

Observou-se que a maioria dos ACS considerou que o seu nível de conhecimento sobre a TB precisa melhorar $(79,17 \%)$ (Tabela 3). É neste contexto que se inserem os ACS, alvos da intervenção educativa. Os achados apontam a relevância de garantir o conhecimento e habilidades específicas para o desempenho das funções desse profissional, pois a qualidade do cuidado fornecida pelo ACS é diretamente influenciada pela sua qualificação (CARDOZO-GONZALES et al., 2015).

Tabela 1. Percepção dos Agentes Comunitários de Saúde quanto ao seu nível de conhecimento sobre a Tuberculose, das duas Unidades de Saúde da Família selecionadas, Porto Velho, 2017

\begin{tabular}{ccc}
\hline Nível de Conhecimento & N=24 & \% \\
\hline Precisa Melhorar & 19 & 79,17 \\
Regular & 1 & 4,17 \\
Bom & 4 & 16,66 \\
Muito Bom & - & - \\
Excelente & - & - \\
\hline
\end{tabular}

Nota: Sinal convencional utilizado: (-) Dado numérico igual a zero não resultante de arredondamento.

A intervenção educativa foi desenvolvida com os ACS das equipes das USF do município, selecionadas para a primeira etapa da pesquisa. Os conteúdos trabalhados foram os identificados, na coleta de dados, com menor percentual de acerto dos entrevistados, sendo: características gerais da TB, diagnóstico, tratamento, medidas de prevenção, vigilância epidemiológica, incluindo a definição de
SR; o controle dos contatos e a notificação dos casos; e, por fim, ILTB.

Os encontros seguiram a programação proposta no planejamento inicial, com apresentação individual, dinâmica de grupo, discussão de texto, exposição dialogada e uma avaliação sobre a intervenção. Todos os participantes empregaram o termo "ótima" para avaliar a metodologia utilizada.

Buscou-se a realização de uma intervenção através de estratégias pedagógicas que estimulassem a participação dos ACS, a apreensão de conteúdo e que considerasse o conhecimento prévio destes profissionais. Além de proporcionar a reflexão e o aprendizado sobre a contribuição do trabalho em equipe e a necessidade de cooperação.

Acredita-se que a resistência dos ACS quanto às atribuições esteja relacionada à falta de conhecimento desses profissionais, não apenas sobre seu papel nas ações de saúde, mas também quanto à falta de conhecimento técnico para o desenvolvimento de tais atividades, e ainda quanto ao sentimento de despreparo advindo de auto constatação, o qual pode ser um fator contribuinte para o processo de qualificação, na perspectiva da educação permanente.

Nesse sentido, é importante ressaltar que a proposta do presente estudo apresentou limitações, uma vez que se trata de uma intervenção pontual, havendo a necessidade de continuidade das atividades educativas, considerando que o controle da TB exige o envolvimento contínuo do ACS em vários aspectos da assistência à saúde.

Nessa perspectiva, investigações futuras que avaliem a existência, bem como a qualidade e a completude das informações referentes ao acompanhamento do tratamento dos doentes de TB na APS do município, serão importantes para a identificação de futuras intervenções com vistas à produção de informações fidedignas. 
Tabela 2. Conhecimento sobre TB dos ACS das duas USF selecionadas, Porto Velho, 2017

\begin{tabular}{|c|c|c|c|c|c|c|c|}
\hline \multirow{2}{*}{ Seção } & \multirow{2}{*}{ Variáveis } & \multicolumn{2}{|c|}{ Acertos } & \multicolumn{2}{|c|}{ Erros } & \multirow{2}{*}{ Categorização } & \multirow{2}{*}{ Achados Importantes } \\
\hline & & n & $\%$ & n & $\%$ & & \\
\hline \multirow{6}{*}{ 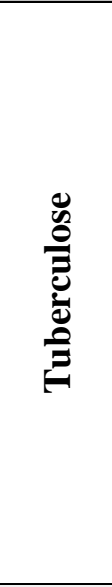 } & $\begin{array}{l}\text { Causa da TB } \\
\text { Bacilo de Koch }\end{array}$ & 4 & 16,67 & 20 & 83,33 & Não adequada & $\begin{array}{l}\text { Infecções respiratórias } \\
\text { Hereditariedade }\end{array}$ \\
\hline & $\begin{array}{l}\text { Sintomas da TB } \\
\text { Tosse por três semanas ou mais + dois outros sintomas (febre, sudorese noturna, } \\
\text { inapetência e emagrecimento) }\end{array}$ & 2 & 8,33 & 22 & 91,67 & Insuficiente & $\begin{array}{l}\text { Dor nas articulações } \\
\text { Vômito } \\
\text { Dor nas costas }\end{array}$ \\
\hline & $\begin{array}{l}\text { Como é transmitida a TB } \\
\text { Via aérea/ar }\end{array}$ & 17 & 70,83 & 7 & 29,17 & Adequada & Através de utensílios \\
\hline & $\begin{array}{l}\text { O que é um contato de TB } \\
\text { Pessoa que convive com portador de TB }\end{array}$ & 4 & 16,67 & 20 & 83,33 & Não adequada & $\begin{array}{l}\text { Pessoas que convivem com } \\
\text { o Tuberculoso }\end{array}$ \\
\hline & $\begin{array}{l}\text { Necessário notificar os casos } \\
\quad \text { Sim }\end{array}$ & 24 & 100,00 & - & - & Adequada & \\
\hline & $\begin{array}{l}\text { Quem notifica } \\
\text { Profissionais de saúde }\end{array}$ & - & - & 24 & 100,00 & Não adequada & SEMUSA \\
\hline \multirow{3}{*}{ 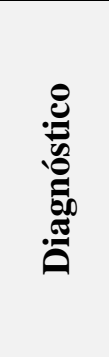 } & $\begin{array}{l}\text { Como é feito } \\
\text { Exame clínico + exames diagnósticos }\end{array}$ & 2 & 8,33 & 22 & 91,67 & Não adequada & Feito no CEMETRON \\
\hline & $\begin{array}{l}\text { Quais os exames realizados } \\
\text { Baciloscopia de escarro + exame radiológico }\end{array}$ & 13 & 54,17 & 11 & 45,83 & Suficiente & $\begin{array}{l}\text { Exames de sangue } \\
\text { Tomografia } \\
\text { Computadorizada }\end{array}$ \\
\hline & $\begin{array}{l}\text { Como coletar o escarro } \\
\text { Local aberto + coletor descartável + pela manhã + não escovar os dentes + escarrar } \\
\text { três vezes no coletor }\end{array}$ & - & - & 24 & 100,00 & Insuficiente & $\begin{array}{l}\text { A enfermeira que coleta } \\
\text { Colocar o dedo na garganta } \\
\text { para escarrar }\end{array}$ \\
\hline \multirow{5}{*}{ 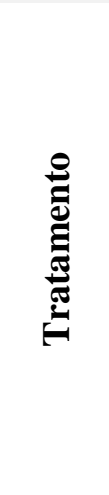 } & $\begin{array}{l}\text { Como se cura a TB } \\
\text { Tratamento completo }\end{array}$ & 10 & 41,67 & 14 & 58,33 & Não adequada & Fazendo o tratamento \\
\hline & $\begin{array}{l}\text { Dose diária da medicação } \\
\text { Uma vez ao dia }\end{array}$ & 4 & 16,67 & 20 & 83,33 & Não adequada & $\begin{array}{l}\text { Depende da conduta do } \\
\text { médico } \\
\text { Não lembro/Não sei }\end{array}$ \\
\hline & $\begin{array}{l}\text { Duração do tratamento da TB } \\
\text { Seis meses }\end{array}$ & 17 & 70,83 & 7 & 29,17 & Adequada & Dois anos \\
\hline & $\begin{array}{l}\text { Tratamento interrompe a transmissão } \\
\text { Sim }\end{array}$ & 23 & 95,83 & 1 & 4,17 & Adequada & Não \\
\hline & $\begin{array}{l}\text { Tempo de transmissão durante tratamento } \\
\text { Aproximadamente } 15 \text { dias }\end{array}$ & 3 & 12,50 & 21 & 87,50 & Não adequada & Depois da primeira dose \\
\hline
\end{tabular}




\begin{tabular}{|c|c|c|c|c|c|c|}
\hline $\begin{array}{l}\text { Tratamento Diretamente Observado } \\
\text { Medicação assistida + profissional de saúde }\end{array}$ & 7 & 29,17 & 17 & 70,83 & Insuficiente & $\begin{array}{l}\text { Acompanhamento de } \\
\text { doente em internação }\end{array}$ \\
\hline $\begin{array}{l}\text { Abandono } \\
30 \text { dias sem comparecer a USF ou sem medicação }\end{array}$ & 2 & 8,33 & 22 & 91,67 & Não adequada & $\begin{array}{l}\text { Doente não encontrado na } \\
\text { residência }\end{array}$ \\
\hline $\begin{array}{l}\text { Conduta em casos de abandono } \\
\text { Busca ativa + retratamento }\end{array}$ & 1 & 4,17 & 23 & 95,83 & Insuficiente & Notificar a SEMUSA \\
\hline $\begin{array}{l}\text { Medidas de prevenção } \\
\text { Orientar o doente a cobrir boca e nariz ao tossir ou espirrar + orientar o doente a usar } \\
\text { máscara + recomendar que doente faça o tratamento }\end{array}$ & - & - & 24 & 100,00 & Insuficiente & $\begin{array}{l}\text { Separar utensílios do doente } \\
\text { Evitar contato com doente }\end{array}$ \\
\hline $\begin{array}{l}\text { Vacina BCG } \\
\text { Prevenir a TB }\end{array}$ & 19 & 79,17 & 5 & 20,83 & Adequada & Prevenir o Tétano \\
\hline $\begin{array}{l}\text { ILTB } \\
\text { Indivíduo bacilífero sem doença ativa }\end{array}$ & 1 & 4,17 & 23 & 95,83 & Não adequada & $\begin{array}{l}\text { TB transmitida da mãe para } \\
\text { o filho }\end{array}$ \\
\hline $\begin{array}{l}\text { Existe quimioprofilaxia para TB } \\
\text { Sim }\end{array}$ & 6 & 25,00 & 18 & 75,00 & Não adequada & Não sei \\
\hline $\begin{array}{l}\text { Quem deve receber a quimioprofilaxia } \\
\text { RN contato de bacilífero + criança não vacinada com BCG + criança vacinada a mais de } \\
\text { dois anos + criança com qualquer condição de imunossupressão + adolescentes/adultos } \\
\text { com ILTB }\end{array}$ & - & - & 24 & 100,00 & Não adequada & Doente de TB \\
\hline $\begin{array}{l}\text { Como é realizada a quimioprofilaxia } \\
\text { Isoniazida }\end{array}$ & - & - & 24 & 100,00 & Não adequada & Não sei \\
\hline $\begin{array}{l}\text { Duração } \\
\text { RN por três meses, podendo ser prorrogado por mais três + adolescente/adulto por no } \\
\text { mínimo seis meses ou } 180 \text { doses }\end{array}$ & - & - & 24 & 100,00 & Não adequada & Não sei \\
\hline
\end{tabular}

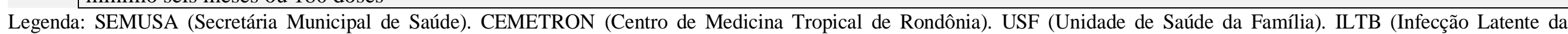
Tuberculose). RN (recém-nascido).

Nota: Sinal convencional utilizado: (-) Dado numérico igual a zero não resultante de arredondamento. 


\section{CONCLUSÃO}

Os resultados do estudo mostraram que a maioria dos ACS entrevistados possuía um conhecimento restrito sobre a TB, além de equívocos sobre a forma de transmissão da doença, o que pode comprometer sobremaneira o alcance das metas estabelecidas para a eliminação da TB como problema de saúde pública até o ano de 2035.

Evidencia-se que os processos educativos têm se restringido à reprodução do conhecimento e à transmissão de conteúdo, desconsiderando os ACS como sujeitos ativos no processo de aprendizagem, dotados de conhecimentos e valores.

Identificou-se a necessidade de que as ações educativas desenvolvidas valorizem a prática diária desses profissionais, numa articulação entre teoria e prática, em que o conhecimento seja construído na realidade vivenciada pelos ACS, para permitir a participação ativa no processo de qualificação. Além disso, faz-se necessário que seja oferecido o Curso de Formação Inicial antes da atuação prática.

Considerando que a qualificação tem um papel central na dinâmica das ações desenvolvidas no trabalho do ACS, cabem reflexões para (re)pensar ações de educação permanente em saúde contínua, crítico-reflexivas, com metodologias e abordagens pedagógicas apropriadas para serem desenvolvidas nos processos educativos.

Estudos como este, ainda que apresentem uma realidade local, podem gerar reflexões sobre a importância da atuação qualificada do ACS no controle da TB e contribuir como ferramenta de gestão, para avaliação das necessidades dos profissionais para educação, além de possibilitar um planejamento coerente e participativo com os atores envolvidos.

\section{AGRADECIMENTO}

Agradecemos à coordenação municipal do Programa de Controle da TB, ao Grupo de Estudos em TB (GET) da Universidade Federal de Rondônia pelo apoio na coleta de dados, e aos profissionais de saúde das duas unidades de saúde, que participaram e permitiram a realização deste estudo.

Todos os autores declararam não haver qualquer potencial conflito de interesses referente a este artigo.

\section{REFERÊNCIAS}

BORNSTEIN, V. J.; DAVID, H. M. S. L. Contribuições da formação técnica do agente comunitário de saúde para o desenvolvimento do trabalho da equipe Saúde da Família. Trab. Educ. Saúde, v. 12, n. 1, p. 107-128, 2014.

BRASIL. Ministério da Saúde. Conselho Nacional de Saúde. Resolução no 466, de 12 de dezembro de 2012. Disponível em: http://bvsms.saude.gov.br/bvs/saudelegis/cns/2013/re s0466_12_12_2012.html. Acesso em 25 out. 2019

BRASIL. Ministério da Saúde. Secretaria de Vigilância em Saúde. Boletim Epidemiológico Tuberculose. Brasília, v. 50, n. 9, março. 2019

BRASIL. Ministério da Saúde. Secretaria de Vigilância em Saúde. Departamento de Vigilância Epidemiológica. Manual de Recomendações para o controle da Tuberculose no Brasil. Brasília: Ministério da Saúde, 2011a.

BRASIL. Ministério da Saúde. Secretaria de Vigilância em Saúde. Departamento de Vigilância Epidemiológica. Tratamento Diretamente Observado da Tuberculose na Atenção Básica: protocolo de enfermagem. Brasília: Ministério da Saúde, $2011 b$.

BRASIL. Ministério da Saúde. Secretaria de Vigilância em Saúde. Departamento de Vigilância das Doenças Transmissíveis. Plano Nacional pelo Fim da Tuberculose. Brasília: Ministério da Saúde, 2017.

CARDOZO-GONZALES, R. I. HARTER, J.; COSTA, L. M.; PINHO, L. B.; LIMA, L. M.; REIS, S. P. A descoberta da Tuberculose no território: análise qualitativa do trabalho do Agente Comunitário de 
Saúde. Ciencia y Enfermeria, v. 21, n. 2, p. 87-97, 2015.

CLEMENTINO, F. S.; MIRANDA, F. A. N. Tuberculose: acolhimento e informação na perspectiva da visita domiciliária. Revista Enfermagem UERJ, v. 23, n. 3, p. 350-354, 2015.

CRISPIM, J. A.; SCATOLIN, B. E.; SILVA, L. M. C.; PINTO, I. C.; PALHA, P. F.; ARCÊNCIO, R. A. Agente Comunitário de Saúde no controle da tuberculose na Atenção Primária à Saúde. Acta Paul. Enferm., v. 25, n. 5, p. 721-727, 2012.

DENTI, I. A.; TOZZO, M. B.; MENDES, L. M. P. Perfil e necessidades básicas de educação dos agentes comunitários de saúde do alto Uruguai, Revista Perspectiva, v. 38, n. [esp.], p. 61-71, 2014.

DURÃO, A. V. R.; MOROSINI, M. V. G. C.; CARVALHO, V. Os agentes comunitários de saúde e o conceito de comunidade na configuração de sua qualificação. In: VIEIRA, M.; DURÃO, A. V. R.; LOPES, M. R. (Orgs.). Para além da comunidade: trabalho e qualificação dos Agentes Comunitários de Saúde. Rio de Janeiro: EPSJV, 2011. p. 119-160.

FRANCO, T. B.; MERHY, E. E. Programa de Saúde da Família (PSF): contradições de um programa destinado à mudança do modelo tecnoassistencial. In: MERHY, E. E. et al. (Orgs.). O trabalho em saúde: olhando e experienciando o SUS no cotidiano. 4 ed. São Paulo: Hucitec, 2007. p.55-124.

FREITAS, L. M.; MARINUS, M. W.; LIMA, L. S.; RUIZ-MORENO, L. Formação dos agentes comunitários de saúde no município de Altamira (PA), Brasil. ABCS Health Sciences, v. 40, n. 3, p. 171-177, 2015.

GARCIA, A. C. P; LIMA, R. C. D.; GALAVOTE, H. S.; COELHO, A. P. S.; VIEIRA, E. C. L.; SILVA, R. C.; ANDRADE, M. A. C. Agente comunitário de saúde no Espírito Santo: do perfil às atividades desenvolvidas. Trabalho, Educação e Saúde, v. 15, n. 1, p. 283-300, 2017.

GOUVÊA, G. R.; SILVA, M. A. V.; PEREIRA, A. C.; MIALHE, F. L.; CORTELLAZZI, K. L.; GUERRA, L. M. Avaliação do conhecimento em saúde bucal de agentes comunitários de saúde vinculados à Estratégia Saúde da Família. Ciência \& Saúde Coletiva, v. 20, n. 4, p. 1185-1197, 2015.

GUIMARÃES, M. S. A.; SOUSA, M. F.; MUCARI, T. B. Perfil sociodemográfico dos agentes comunitários de saúde da estratégia saúde da família no município de Palmas-TO. Desafios, v. 4, n. 3, p. 6072, 2017.

LANZONI, G. M. M.; CECHINEL, C.; MEIRELLES, B. H. S. Agente Comunitário de Saúde: estratégias e consequências da sua rede de relações e interações. Rev. RENE, v. 15, n. 1, p. 123-131, 2014.

LIMA， L. M.; SCHWARTZ, E. CARDOZOGONZALES, R. I. HARTER, J.; LIMA, J. F. O programa de controle da tuberculose em Pelotas/RS, Brasil: investigação de contatos intradomiciliares. Rev. Gaúcha Enferm., v. 34, n. 2, p. 102-110, 2013.

MELO, M. B.; QUINTÃO, A. F.; CARMO, R. F. O Programa de Qualificação e Desenvolvimento do Agente Comunitário de Saúde na perspectiva dos diversos sujeitos envolvidos na atenção primária em saúde. Saúde e Sociedade, v. 24, n. 1, p. 86-99, 2015.

QUEIROZ, D. M.; SILVA, M. R. F.; OLIVEIRA, L. C. Educação Permanente com Agentes Comunitários de Saúde: potencialidades de uma formação norteada pelo referencial da Educação Popular e Saúde. Interface-Comunicação, Saúde, Educação, v. 18, supl. 2, p. 1199-1210, 2014.

SÁ, L. D.; ANDRADE, M. N.; NOGUEIRA, J. A.; VILLA, T. C. S.; FIGUEIREDO, T. M. R. M. QUEIROGA, R. P. F.; SOUSA, M. C. M. Implantação da estratégia DOTS no controle da Tuberculose na Paraíba: entre o compromisso político e o envolvimento das equipes do Programa Saúde da Família (1999-2004). Ciência \& Saúde Coletiva, v. 16, n. 9, p. 3917-3924, 2011.

SOUZA, C. F.; BEN, A. J.; SCHNEIDER, S. M. B.; NASCIMENTO, B. P.; NEUMANN, C. R.; OLIVEIRA, F. J. A. Q. A importância das ações programáticas de saúde no controle da tuberculose: experiência de um serviço de atenção primária à saúde em Porto Alegre, Rio Grande do Sul, Brasil. Clinical and Biomedical Research., Porto Alegre, v. 34, n. 2, p. 175-183, 2014.

TOMAZ, J. B. C. O agente comunitário de saúde não deve ser um "super-herói". Interface, v. 6, n. 10, p. 8487, 2002.

VILLA, T. C. S.; PONCE, M. A. Z.; WYSOCKI, A. D.; ANDRADE, R. L. P.; ARAKAWA, T.; SCATOLIN, B. E.; BRUNELLO, M. E. F.; BERALDO, A. A.; SCATENA, L. M.; MONROE, A. A.; SOBRINHO, R. A. S.; SÁ, L. D.; NOGUEIRA, J. A.; ASSIS, M. M. A.; CARDOZO-GONZALES, R. I.; PALHA, P. F. Diagnóstico oportuno da tuberculose nos serviços de saúde de diversas regiões do Brasil. 
Rev. Latino-Am. Enfermagem, v. 21, n. [esp.], p. 190-198, 2013.

ORGANIZAÇÃO MUNDIAL DE SAÚDE (OMS). Advocacia, Comunicação e Mobilização Social para o Controle da TB: Um Guia para o
Desenvolvimento de Pesquisas de Conhecimento, Atitudes e Práticas. 2008

WORLD HEALTH ORGANIZATION (WHO). Global Tuberculosis Report 2019. Geneva: WHO, 2019. 